\title{
Defining the optimal target for anterior thalamic deep brain stimulation in patients with drug-refractory epilepsy
}

\author{
*Wendy Guo, ${ }^{1}$ Bang-Bon Koo, PhD,1 Jae-Hun Kim, PhD, ${ }^{2}$ Rafeeque A. Bhadelia, MD, ${ }^{3}$ \\ Dae-Won Seo, MD, ${ }^{4}$ Seung Bong Hong, MD, ${ }^{4}$ Eun Yeon Joo, MD, ${ }^{4}$ Seunghoon Lee, MD, ${ }^{5}$ \\ Jung-II Lee, MD, ${ }^{5}$ Kyung Rae Cho, MD, ${ }^{5}$ and Young-Min Shon, MD ${ }^{4,6}$
}

\begin{abstract}
'Department of Anatomy and Neurobiology, Boston University School of Medicine, Boston, Massachusetts; '2Department of Radiology, Samsung Medical Center, Sungkyunkwan University School of Medicine, Seoul, Republic of Korea; ${ }^{3}$ Department of Radiology, Beth Israel Deaconess Medical Center, Harvard Medical School, Boston, Massachusetts; ${ }^{4}$ Department of Neurology, Samsung Medical Center, Sungkyunkwan University School of Medicine, Seoul; ${ }^{5}$ Department of Neurosurgery, Samsung Medical Center, Sungkyunkwan University School of Medicine, Seoul; and ${ }^{6}$ Department of Health Sciences and Technology, SAIHST, Sungkyunkwan University, Seoul, Republic of Korea
\end{abstract}

\begin{abstract}
OBJECTIVE The anterior thalamic nucleus (ATN) is a common target for deep brain stimulation (DBS) for the treatment of drug-refractory epilepsy. However, no atlas-based optimal DBS (active contacts) target within the ATN has been definitively identified. The object of this retrospective study was to analyze the relationship between the active contact location and seizure reduction to establish an atlas-based optimal target for ATN DBS.

METHODS From among 25 patients who had undergone ATN DBS surgery for drug-resistant epilepsy between 2016 and 2018, those who had follow-up evaluations for more than 1 year were eligible for study inclusion. After an initial stimulation period of 6 months, patients were classified as responsive ( $\geq 50 \%$ median decrease in seizure frequency) or nonresponsive ( $<50 \%$ median decrease in seizure frequency) to treatment. Stimulation parameters and/or active contact positions were adjusted in nonresponsive patients, and their responsiveness was monitored for at least 1 year. Postoperative CT scans were coregistered nonlinearly with preoperative MR images to determine the center coordinate and atlasbased anatomical localizations of all active contacts in the Montreal Neurological Institute (MNI) 152 space.
\end{abstract}

RESULTS Nineteen patients with drug-resistant epilepsy were followed up for at least a year following bilateral DBS electrode implantation targeting the ATN. Active contacts located more adjacent to the center of gravity of the anterior half of the ATN volume, defined as the anterior center (AC), were associated with greater seizure reduction than those not in this location. Intriguingly, the initially nonresponsive patients could end up with much improved seizure reduction by adjusting the active contacts closer to the $\mathrm{AC}$ at the final postoperative follow-up.

CONCLUSIONS Patients with stimulation targeting the AC may have a favorable seizure reduction. Moreover, the authors were able to obtain additional good outcomes after electrode repositioning in the initially nonresponsive patients. Purposeful and strategic trajectory planning to target this optimal region may predict favorable outcomes of ATN DBS. https://thejns.org/doi/abs/10.3171/2020.2.JNS193226

KEYWORDS deep brain stimulation; drug-refractory epilepsy; anterior thalamic nuclei; thalamic parcellation; nonlinear analysis; DISTAL; functional neurosurgery

$\mathrm{E}$ PILEPSY, characterized by recurring, unprovoked seizures, is one of the most common neurological disorders, affecting over 65 million people worldwide. ${ }^{1}$ Antiseizure medications are the most typical form of treatment; however, for patients with intractable seizures who are not candidates for resective surgery, deep brain stimu- lation (DBS) has become a promising therapeutic alternative. One common DBS target is the anterior thalamic nucleus (ATN). Clinically, the effectiveness of ATN DBS for epilepsy has been reported in previous studies showing reductions in seizure frequency. ${ }^{2-5}$ In addition, the anticonvulsant effects of ATN DBS have been shown to be

ABBREVIATIONS AC = anterior center, i.e., the center of gravity of the anterior half of the ATN; AED = antiepileptic drug; $A T N=$ anterior thalamic nucleus; DBS = deep brain stimulation; DISTAL = DBS Intrinsic Template AtLas; EVA = extraventricular approach; IPG = internal pulse generator; MNI = Montreal Neurological Institute; MTT = mammillothalamic tract; R28 = region 28; SyN = symmetric image normalization; TVA = transventricular approach.

SUBMITTED November 29, 2019. ACCEPTED February 24, 2020.

INCLUDE WHEN CITING Published online May 8, 2020; DOI: 10.3171/2020.2.JNS193226.

* W.G. and B.B.K. contributed equally to this work and share first authorship. 
sustained with cognitive improvements. ${ }^{6,7}$ However, there is still variation in the seizure reduction response to ATN DBS across patients and even within responsive outcomes, and it is not clear whether these variations arise from disparities in the stimulation location within the ATN. It is possible that DBS surgeries utilizing indirect anterior commissure-posterior commissure coordinates to target the ATN do not account for the high degree of anatomical variation between patients and likely contribute to variations in stimulation location and thus outcome. ${ }^{8}$

In this study, we utilized a nonlinear, atlas-based approach to investigate the relationship between the active contact (contacts being stimulated) location and the seizure reduction percentage in patients receiving ATN DBS. Nonlinear registrations of postoperative CT scans and preoperative MR images to the Montreal Neurological Institute (MNI) 152 space were followed by atlas-based localization of active contacts. First, we determined whether there were differences in the MNI coordinates of active contacts between responders and nonresponders after ATN DBS. Second, we investigated whether there was an optimal site for active contacts within the ATN that was associated with more favorable outcomes.

\section{Methods \\ Patients}

This retrospective study was conducted with the approval of the Institutional Review Board of the Samsung Medical Center (Seoul, Republic of Korea), and informed written consent had been obtained from all patients or their family members at the time of treatment. From among 25 patients who had undergone ATN DBS surgery for drug-resistant epilepsy between 2016 and 2018, those who had follow-up evaluations for more than 1 year were eligible for this study. Follow-up assessments were conducted during routine outpatient clinic visits (less than 3 months apart) and hospital visits for reoperation or scheduled battery changes.

Our criteria for bilateral ATN DBS device implantation, which have been published elsewhere, ${ }^{6,7}$ were as follows: frequent ( $>4$ per month) and disabling seizures not controlled by multiple antiepileptic drug (AED) treatment modalities, not a candidate for resective surgical treatment as determined by video-EEG monitoring (e.g., multifocal ictal onset zone), failure of previous resective or disconnective surgery, failure of previous vagus nerve stimulation therapy, agreement by patients (or caregivers) to keep a daily seizure diary for a 3-month baseline period before DBS device implantation and continuously after initiation of treatment, and agreement by patients (or caregivers) that no changes to the baseline presurgical medication regimen would be made during at least the 1st year after DBS device implantation.

\section{Surgical Technique and Brain Imaging}

A Leksell model G stereotactic head frame (Elekta Instruments) was applied, and a stereotactic preoperative scan was obtained using a Siemens Trio 3-T scanner (Siemens AG, Healthcare Sector) with a 64-channel head and neck coil. As part of the imaging protocol, T1-weighted
3D magnetization-prepared rapid acquisition gradient echo (MPRAGE), T2-weighted FLAIR, and fast gray matter acquisition T1 inversion recovery (FGATIR) sequences were obtained for targeting purposes.

We targeted the ATN on parasagittal MRI as a prominence in the inferior wall of the lateral ventricle. Using the FGATIR data set, we directly identified the mammillothalamic tract (MTT), and the trajectory was planned to run from the inferior-lateral aspect of the ATN, aiming to stimulate the MTT-ATN junction. ${ }^{9}$

The transventricular insertion of DBS electrodes (model 3389 [ $n=14]$ or 3387 [ $n=5$ ], Medtronic Inc.) and subsequent implantation ( 2 or 3 days after electrode insertion) of internal pulse generators (IPGs; Activa PC, Medtronic Inc.) were performed as previously described ${ }^{4,10} \mathrm{~A}$ rigid guide cannula was inserted and advanced deep into the brain to a point $5 \mathrm{~mm}$ from the desired target under direct fluoroscopic and Leksell frame guidance. Microelectrode recordings were performed to identify the neural signals obtained from the ATN and dorsal medial nucleus.

Finally, an intraoperative CT scan was obtained with the patient's head still fixed in the stereotactic head ring. The CT scan was fused to the initial preoperative MR image, and the precision of the fusion images was confirmed using FrameLink software (Medtronic Inc.).

\section{Selection of the Initial Stimulation Lead and Stimulation Parameters}

The location of the initial electrical stimulation lead was determined to be where the maximal driving response (rhythmic theta slow activity caused by stimulation of $5 \mathrm{~Hz}, 5-8 \mathrm{~V}$, and 180-270 $\mu \mathrm{sec}$ ) was recorded on scalp EEG. ${ }^{11}$ During 5-Hz stimulation, the maximal and consistent rhythmic theta activity, time-locked to stimulation, should be observed at the selected DBS lead. One or 2 weeks later, activation and programming of the IPGs was started. The initial chronic stimulation parameters were similar to the parameters used for surgery for movement disorders (high frequency of $130 \mathrm{~Hz}$, pulse width of 90 usec, continuous stimulation). With the aid of a previously reported programming algorithm, ${ }^{6,7}$ the relatively lowvoltage (1.5-3.1 V) stimulation and monopolar configuration could be adjusted after the 6th postoperative month for optimizing therapeutic efficacy and minimizing the side effects of individual patients.

\section{Evaluation of Clinical Outcome}

Long-term follow-up and adjustment of DBS parameters were conducted (Y.M.S., E.Y.J., D.W.S.). Retrospective chart review was performed to collect follow-up and outcome data. All patients had the opportunity to have received DBS therapy for at least 1 year. Data collected from the last available clinical follow-up were as follows: mean weekly seizure frequency (from seizure diaries kept by patients or caregivers), adverse effects of DBS therapy, stimulation parameters, changes in AED regimens (if they existed), and timing of dead IPG replacement.

We categorized patients as responders $(\geq 50 \%$ median decrease in seizure frequency for each 3-month period as compared to the preimplant baseline) or nonresponders 


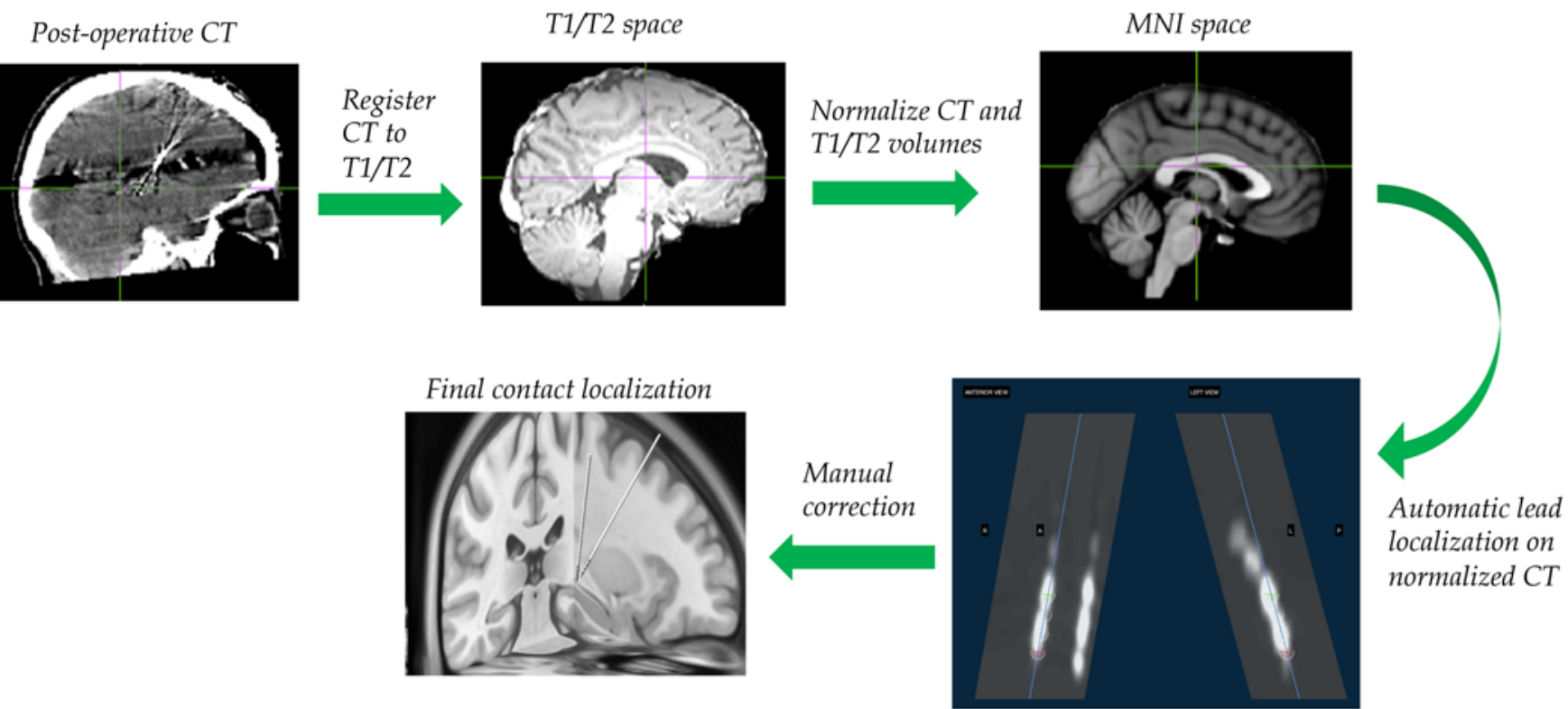

FIG. 1. Lead-DBS processing to determine active contact coordinate and anatomical localization. With the aid of Lead-DBS, postoperative CT and preoperative MR images were normalized to MNI space, followed by lead localization using electrode artifacts. Figure is available in color online only.

( $<50 \%$ median decrease in seizure frequency). The sources of the data included outpatient clinic visits, medical record review, and telephone interviews. To limit the bias created by nonresponder attrition, we computed follow-up duration using a last observation carried forward (LOCF) analysis instead of a declining-n analysis. ${ }^{12}$ In addition, we included only those patients who had at least 1 year of follow-up after initiation of ATN DBS and reliable documentation of long-term seizure frequency.

\section{Active Contact Coordinate Determination}

Image processing and active contact localization were performed using Lead-DBS (Fig. 1), a MatLab toolbox, with statistical parametric mapping 12 (SPM12). ${ }^{13}$ Based on advanced normalization tools (ANTs), a rigid registration followed by an affine one was used to linearly register the post- and intraoperative CT to the preoperative MRI. ${ }^{14}$ Symmetric image normalization (SyN) registration in the ANTs was used to spatially normalize preand postoperative scans into MNI ICBM 2009b nonlinear asymmetrical space based on preoperative MRI. ${ }^{14,15}$ Nonlinear transformation into template space composed of two linear (rigid followed by affine) registrations, a whole-brain nonlinear SyN registration, and two nonlinear SyN registrations focused on the subcortical area. ${ }^{14,16}$ Electrode localizations were corrected for brain shift on postoperative CT by applying a refined affine transform calculated between pre- and postoperative scans. ${ }^{13}$ The Precise and Convenient Electrode Reconstruction for Deep Brain Stimulation (PaCER) algorithm was used to localize electrodes in native and template space. ${ }^{17}$ Electrode localizations were then visually inspected and manually corrected if needed. The manual correction was performed based on postoperative CT by placing the trajectory as precisely as possible within the center of the electrode artifact. ${ }^{13}$

Based on electrode localization and contact spacing, the center MNI coordinate for each active contact was determined. More positive $\mathrm{x}-, \mathrm{y}$-, and $\mathrm{z}$-coordinates represent more rightward, anterior, and superior locations, respectively. Image analysis was performed by two authors (W.G., B.B.K.) blinded to the outcome of DBS therapy.

\section{Anatomical Localization of the Active Contact}

In MNI space, specific localization of the active contacts to anatomical subcortical structures was manually determined in the DBS Intrinsic Template AtLas (DISTAL). Region 28 (R28) in the DISTAL template covers most areas of the ATN (Fig. 2), including the nucleus anterior principalis and nucleus anteromedialis in the Hassler/ Schaltenbrand-Wahren nomenclature, ${ }^{18,19}$ and was chosen as the region of interest for assessing active contact localization for ATN DBS in each patient.

\section{Statistical Analysis}

The MNI x-, y-, and z-coordinates of active contacts were separately analyzed using t-tests between responsive and nonresponsive patients to determine whether significant differences along an axis could explain the difference in outcome. A fitted linear regression or quadratic model between the active contact coordinates along each axis and seizure reduction was tested.

To further determine the optimal stimulation target for ATN DBS, we determined the Euclidean distance between each active contact in patients and the reference point that represents the central location of the ATN (R28, regions drawn in yellow and orange in Fig. 2). The coordinates 

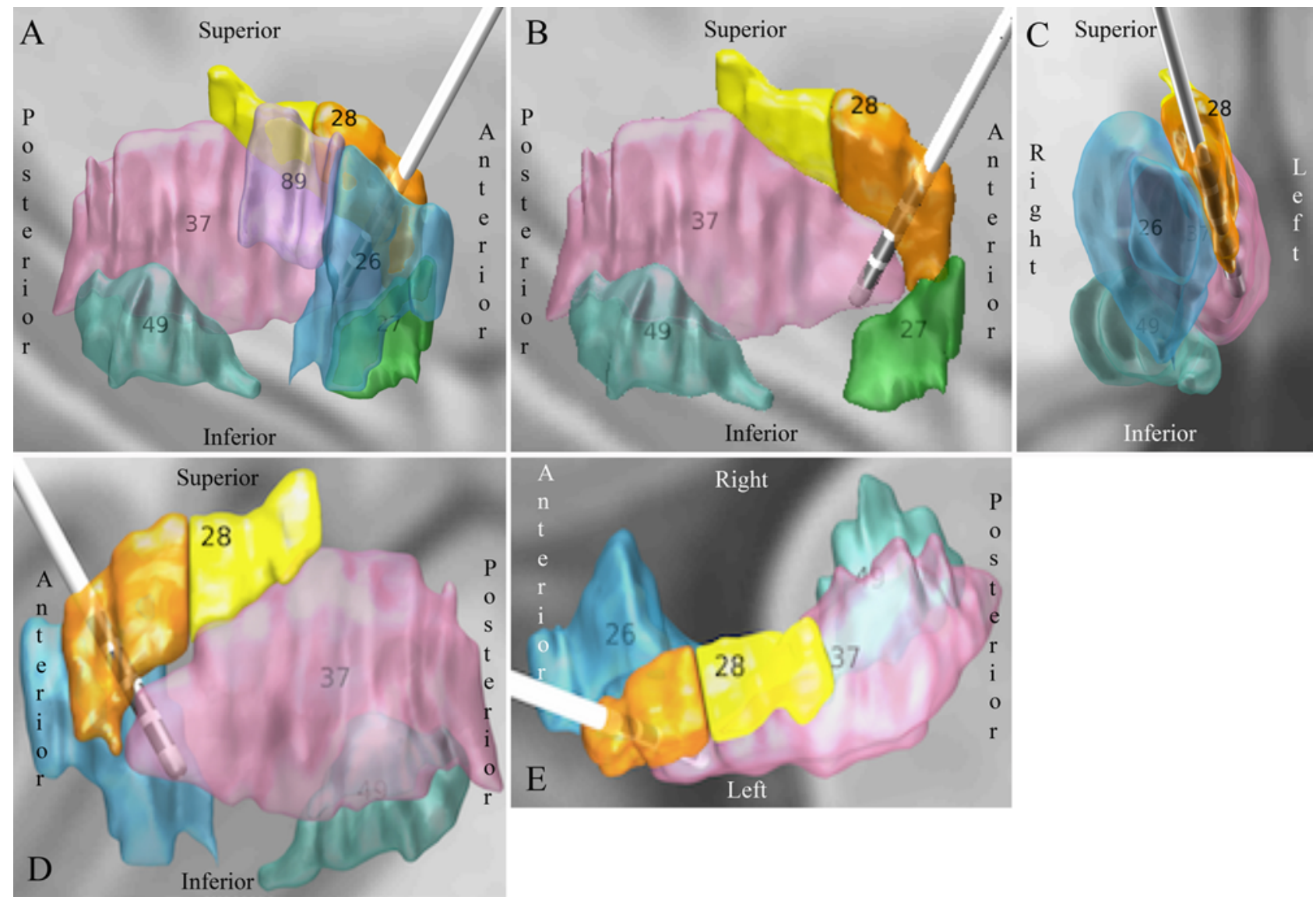

FIG. 2. DISTAL parcellation of the thalamus: $26=$ nucleus lateropolaris thalami; $27=$ nucleus fasciculosus thalami; $28=$ nucleus anterior principalis combined with the nucleus anteromedialis and divided into anterior (orange) and posterior (yellow) regions; 37 = nucleus medialis; 49 = nucleus centralis; 89 = nucleus dorsooralis internus. Figure is available in color online only.

of the ATN surface from the DISTAL were averaged to obtain the overall center coordinate for each hemisphere. However, as active contacts were mostly localized within the anterior half of the ATN, the center of gravity of the anterior half of the ATN (orange portion of R28 in Fig. 2), defined as the anterior center (AC), must be a more appropriate reference point in comparison with the overall center for each hemisphere. Fitted linear regression models between distance and seizure reduction were tested for all final active contacts and additional active contacts.

\section{Results}

\section{Clinical Response to ATN DBS}

Nineteen patients ( 9 females and 10 males, with a mean age of $34.8 \pm 9.9$ years [mean \pm standard deviation]) were included in the final analysis. Six patients were excluded because of a follow-up $<1$ year $(n=3)$, a target location other than the ATN $(\mathrm{n}=1)$, unreliable clinical information on seizure frequency $(n=1)$, and an implant site infection leading to removal of the DBS devices $(n=1)$. The mean duration of epilepsy before DBS surgery was $19.3 \pm 9.0$ years, most patients were undergoing AED polytherapy (4.6 \pm 2.7 regimens), and several AEDs (5.2 \pm 4.1 drugs) had failed in most patients in the past. The mean followup duration of the ATN DBS trials was $23.8 \pm 7.5$ months (Table 1).

\section{Seizure Outcome and Follow-Up Data}

Following the initial stimulation for 6 months, $9(47.4 \%)$ patients had been responsive and received no further changes to their active contact positions. The remaining 10 nonresponsive patients had changes in their stimulation parameters and/or active contact positions after the first 6 months of follow-up. Among the nonresponders, 2 patients were switched from monopolar to bipolar stimulation at the initial one contact (case 12) or two (case 16) because of high impedance at one of the active electrodes. Another 2 (10.5\%) nonresponders with initial stimulation in one monopolar contact made a change into activating the other two adjacent electrodes and remained under monopolar stimulation (cases 13 and 15). The 6 (31.6\%) remaining initial nonresponders received stimulation to an additional contact pair and remained under monopolar stimulation (Table 2).

Six $(60 \%)$ of the 10 initially nonresponsive patients eventually became responders after judicious tuning of their active contacts; therefore, the overall responder rate 
TABLE 1. Demographics of the patients with ATN DBS

\begin{tabular}{|c|c|c|c|c|c|c|c|c|}
\hline Case No. & Sex & Age (yrs) & Postop FU (mos) & Final Response & Epilepsy Syndrome & Etiological Lesions & Previous Hx of VNS & Electrode Model \\
\hline 1 & $M$ & 41 & 42 & Yes & FLE & UK & & 3389 \\
\hline 2 & $\mathrm{~F}$ & 62 & 29 & Yes & TLE & Bilat HS & & 3389 \\
\hline 3 & $M$ & 36 & 16 & Yes & F-TLE & Encephalitis & & 3389 \\
\hline 4 & M & 50 & 17 & Yes & TLE & Bilat HS & & 3389 \\
\hline 5 & M & 26 & 19 & Yes & F-TLE & FCD \& PMG & & 3387 \\
\hline 6 & $\mathrm{~F}$ & 22 & 15 & Yes & TLE & Lt HS & & 3389 \\
\hline 7 & M & 41 & 18 & Yes & TLE & Bilat HS & & 3389 \\
\hline 8 & $\mathrm{~F}$ & 27 & 22 & Yes & F-TLE & UK & Yes & 3389 \\
\hline 9 & $M$ & 42 & 13 & Yes & TLE & Bilat HS & & 3389 \\
\hline 10 & M & 41 & 36 & Yes & MFE & T-EM & & 3389 \\
\hline 11 & $\mathrm{~F}$ & 39 & 23 & Yes & MFE & FCD & Yes & 3387 \\
\hline 12 & $M$ & 32 & 35 & Yes & TLE & Bilat HS & & 3389 \\
\hline 13 & M & 34 & 28 & Yes & MFE & Encephalitis & Yes & 3389 \\
\hline 14 & $\mathrm{~F}$ & 30 & 22 & No & MFE & UK & Yes & 3387 \\
\hline 15 & $\mathrm{~F}$ & 29 & 37 & No & MFE & UK & & 3387 \\
\hline 16 & $\mathrm{~F}$ & 27 & 23 & No & F-CLE & FCD & & 3389 \\
\hline 17 & $\mathrm{~F}$ & 28 & 28 & Yes & MFE & Encephalitis & Yes & 3387 \\
\hline 18 & $M$ & 26 & 22 & No & TLE & Encephalitis & & 3389 \\
\hline 19 & $\mathrm{~F}$ & 28 & 31 & Yes & FLE & Encephalitis & Yes & 3389 \\
\hline
\end{tabular}

$\mathrm{FCD}$ = focal cortical dysplasia; F-CLE = fronto-central epilepsy; FLE = frontal lobe epilepsy; F-TLE = frontotemporal lobe epilepsy; FU = follow-up; HS = hippocampal sclerosis; $\mathrm{Hx}$ = history; MFE = multifocal epilepsy; PMG = polymicrogyria; T-EM = traumatic encephalomalacia; TLE = temporal lobe epilepsy; UK = unknown; VNS = vagus nerve stimulation.

was $78.9 \%$ (15 of 19 patients; Table 2). The final average seizure reduction for all patients was $64.3 \%$ (range $0 \%-$ $100 \%$, with SD 27.6\%).

\section{MNI Coordinates of Active Contacts}

Analyzing all final active contacts, we found a significant difference in the mean $\mathrm{z}$-coordinate between responders $($ mean $=9.4967)$ and nonresponders (mean $=6.0115$; $\mathrm{p}<0.01)$. This relationship was further characterized as a positive linear relationship between their z-coordinate and seizure reduction percentage $\left(\mathrm{p}<0.01, \mathrm{R}^{2}=0.174, \mathrm{r}=\right.$ 0.4168; Fig. 3A).

We found a similar relationship when comparing only the $\mathrm{z}$-coordinates of additional active contacts and final seizure reduction in the initial nonresponders $\left(\mathrm{p}<0.01, \mathrm{R}^{2}\right.$ $=0.313, \mathrm{r}=0.5598$; Fig. 3B). In addition, we determined a quadratic relationship between the $\mathrm{z}$-coordinate of the active contact and the seizure reduction outcome for initially responsive patients $(\mathrm{y}=-1.4489 \mathrm{x} 2+28.361 \mathrm{x}-57.512, \mathrm{p}$ $<0.05, \mathrm{R}^{2}=0.201$, data not shown).

However, no significant differences in the $\mathrm{x}$ - and $\mathrm{y}$ coordinates were found, as indicated by the distribution of active contacts in MNI space. There was no significant difference in the $\mathrm{x}$-coordinate of all final active contacts between responders (mean $=5.4152$ ) and nonresponders (mean $=5.6231, \mathrm{p}=0.6061)$. This was also the case when analyzing all initial active contacts between patients who were responsive on first stimulation (mean $=5.4846)$ and patients who were unresponsive on first stimulation (mean $=5.5125, \mathrm{p}=0.4750)$. In addition, we found no signifi- cant difference in the $\mathrm{x}$-coordinate of all additional active contacts between patients who became responsive after changes in stimulation (mean $=5.3636)$ and patients who remained unresponsive $($ mean $=5.2556, \mathrm{p}=0.8622)$.

Similar results were obtained when analyzing the $y$ coordinates. For all final active contacts, responders had a mean of -6.2764 and nonresponders had a mean of -6.8831 ( $p=0.3480)$. For all initial active contacts, there was no significant difference between patients who were responsive on first stimulation (mean $=-6.5583$ ) and patients who were unresponsive on first stimulation (mean $=-6.5265, \mathrm{p}=0.4389)$. For all additional active contacts, we found no significant difference in the $y$-coordinate between patients who became responsive after changes in stimulation (mean $=-6.5314$ ) and those who remained unresponsive $($ mean $=-6.0093, \mathrm{p}=0.6742)$.

\section{Distance Between Active Contacts and the AC}

For each patient, the Euclidean distance between each active contact and the AC was calculated for each hemisphere. The AC was set as our reference center because on visual inspection (Fig. 3C and D), it was clear that the $\mathrm{AC}$ was a more appropriate reference than the overall center.

Analysis of all 58 final active contacts showed a negative linear relationship between distance from active contact to AC and seizure reduction percentage $\left(\mathrm{p}<0.01, \mathrm{R}^{2}\right.$ $=0.217, \mathrm{r}=-0.4660 ;$ Fig. $4 \mathrm{~A})$. Analysis of only additional active contacts in the initial nonresponders revealed a similar linear relationship $\left(\mathrm{p}<0.05, \mathrm{R}^{2}=0.252, \mathrm{r}=-0.5017\right.$; Fig. 4B). Furthermore, we found a negative linear relation- 
TABLE 2. DBS contacts and their related therapeutic responses

\begin{tabular}{cccrccc}
\hline $\begin{array}{c}\text { Case } \\
\text { No. }\end{array}$ & $\begin{array}{c}\text { Initial Active } \\
\text { Contact: Rt/Lt }\end{array}$ & $\begin{array}{c}\text { Final Active } \\
\text { Contact: Rt/Lt }\end{array}$ & $\begin{array}{c}\text { Sz Reduction at } \\
\text { 6 Mos Postop }\end{array}$ & $\begin{array}{c}\text { Final Sz } \\
\text { Reduction }\end{array}$ & $\begin{array}{c}\text { Final Good Outcome } \\
\text { (sz reduction }>50 \%)\end{array}$ & $\begin{array}{c}\text { Adjustment of } \\
\text { DBS Contacts }\end{array}$ \\
\hline 1 & $1 / 1$ & $1 / 1$ & $60.0 \%$ & $50.0 \%$ & Yes & NC \\
\hline 2 & $2 / 2$ & $2 / 2$ & $75.0 \%$ & $66.7 \%$ & Yes & NC \\
\hline 3 & $3 / 2$ & $3 / 2$ & $55.6 \%$ & $64.3 \%$ & Yes & NC \\
\hline 4 & $2 / 2$ & $2 / 2$ & $95.0 \%$ & $88.9 \%$ & Yes & NC \\
\hline 5 & $2 / 3$ & $2 / 3$ & $85.0 \%$ & $96.6 \%$ & Yes & NC \\
\hline 6 & $2 / 2$ & $2 / 2$ & $66.7 \%$ & $70.0 \%$ & Yes & NC \\
\hline 7 & $2 / 2$ & $2 / 2$ & $72.6 \%$ & $62.5 \%$ & Yes & NC \\
\hline 8 & $2 / 2$ & $2 / 2$ & $100.0 \%$ & $100.0 \%$ & Yes & NC \\
\hline 9 & $2 / 2$ & $2 / 2$ & $90.0 \%$ & $100.0 \%$ & Yes & NC \\
\hline 10 & $2 / 2$ & $2,3 / 2,3$ & $44.7 \%$ & $73.5 \%$ & Yes & Yes \\
\hline 11 & $3 / 2$ & $2,3 / 2,3$ & $41.6 \%$ & $88.9 \%$ & Yes & Yes \\
\hline 12 & $1 / 1$ & $1-, 2+/ 1-, 2+$ & $33.3 \%$ & $83.3 \%$ & Yes & Yes \\
\hline 13 & $2 / 2$ & $1,3 / 1,3$ & $44.4 \%$ & $50.0 \%$ & Yes & Yes \\
\hline 14 & $2 / 2$ & $1,2 / 1,2$ & $28.6 \%$ & $28.6 \%$ & No & Yes \\
\hline 15 & $2 / 2$ & $1,3 / 1,3$ & $33.3 \%$ & $25.0 \%$ & No & Yes \\
\hline 16 & $3,1 / 3,1$ & $1+, 3-/ 1+, 3-$ & $-15.0 \%$ & $0.0 \%$ & No & Yes \\
\hline 17 & $2 / 2$ & $1,2 / 1,2$ & $25.0 \%$ & $75.0 \%$ & Yes & Yes \\
\hline 18 & $2 / 2$ & $2,3 / 2,3$ & $25.0 \%$ & $33.0 \%$ & No & Yes \\
\hline 19 & $2 / 2$ & $2,3 / 2,3$ & $40.0 \%$ & $65.0 \%$ & & \\
\hline
\end{tabular}

$\mathrm{NC}=$ no change; $\mathrm{sz}=$ seizure

ship between distance and $\mathrm{z}$-coordinate for not only all final active contacts $\left(\mathrm{p}<0.01, \mathrm{R}^{2}=0.533, \mathrm{r}=-0.7303\right.$; Fig. $5 \mathrm{~A})$ but also the additional stimulated contacts $(\mathrm{p}<0.01$, $\mathrm{R}^{2}=0.634, \mathrm{r}=-0.7964$; Fig. 5B).

\section{Discussion}

In this study, we analyzed the relationship between the location of contacts being stimulated (active contacts) and seizure outcome after ATN DBS therapy for patients with drug-resistant epilepsy. To our knowledge, this is the first study showing the usefulness of a group mapping by nonlinear transformation to take into account the actual 3D surrounding anatomical structures of ATN DBS patients. We specifically assessed whether there is an optimal location within the ATN, where it is not only easily and practically applicable to clinicians but also associated with the best postsurgical outcomes.

\section{Clinical Implication of Localizing Optimal Contacts in ATN DBS Therapy}

We demonstrated that active contacts much closer to the $\mathrm{AC}$ were associated with better outcomes. Moreover, additional contacts toward more superoanterior regions of the ATN in initially nonresponsive patients mostly resulted in a much more favorable outcome after revision of the active contacts. Therefore, the AC may be the optimal stimulation target in ATN DBS for epilepsy.

These findings are in line with previous results using indirect atlas-based targeting with stereotactic coordinates ${ }^{3}$ or the linearly normalized ATN coordinate system.9,21
However, the heterogeneous inclusion criteria for epilepsy syndrome, follow-up periods after stimulation, and stimulation parameters (continuous vs cyclic mode) among those studies make it difficult to reach a convincing conclusion. Validation of our results will require prospective testing.

\section{Practical and Ideal Trajectory for Targeting the ATN}

The ATN is a unique structure with a sequestered location surrounded by the ventricle, making electrode insertion intricate. To date, three methods for a trajectory to the ATN have been tried. The transventricular approach (TVA) is the most commonly used, entering from the frontal area toward the junction between the ipsilateral ATN and MTT, usually targeting about 3.5-4 $\mathrm{mm}$ below the surface of the thalamus. ${ }^{9,10}$ The extraventricular approach (EVA), in contrast, has a much lower entry point without touching deep vessels located at the floor of the lateral ventricle (e.g., choroid plexus and thalamostriatal vein). ${ }^{21,22}$ A very recent study comparing these two methods showed that the TVA offers a significantly higher chance of placing DBS leads in the ATN correctly. ${ }^{23}$ (Compared to DBS leads placed via an EVA, those placed via the TVA were positioned $19 \%$ more correctly on either the left or right hemisphere and $26 \%$ more exactly on both sides.) This result may be concordant with our results considering that TVA is an effective trajectory that enables the upper two leads to be located in the ATN (especially at the AC) because the height of the ATN is about 5-6 mm in humans. The less favorable outcome with the EVA reported by Herrman et al. ${ }^{22}$ seems to be related to the EVA rather than the TVA; however, that study's small patient population and 

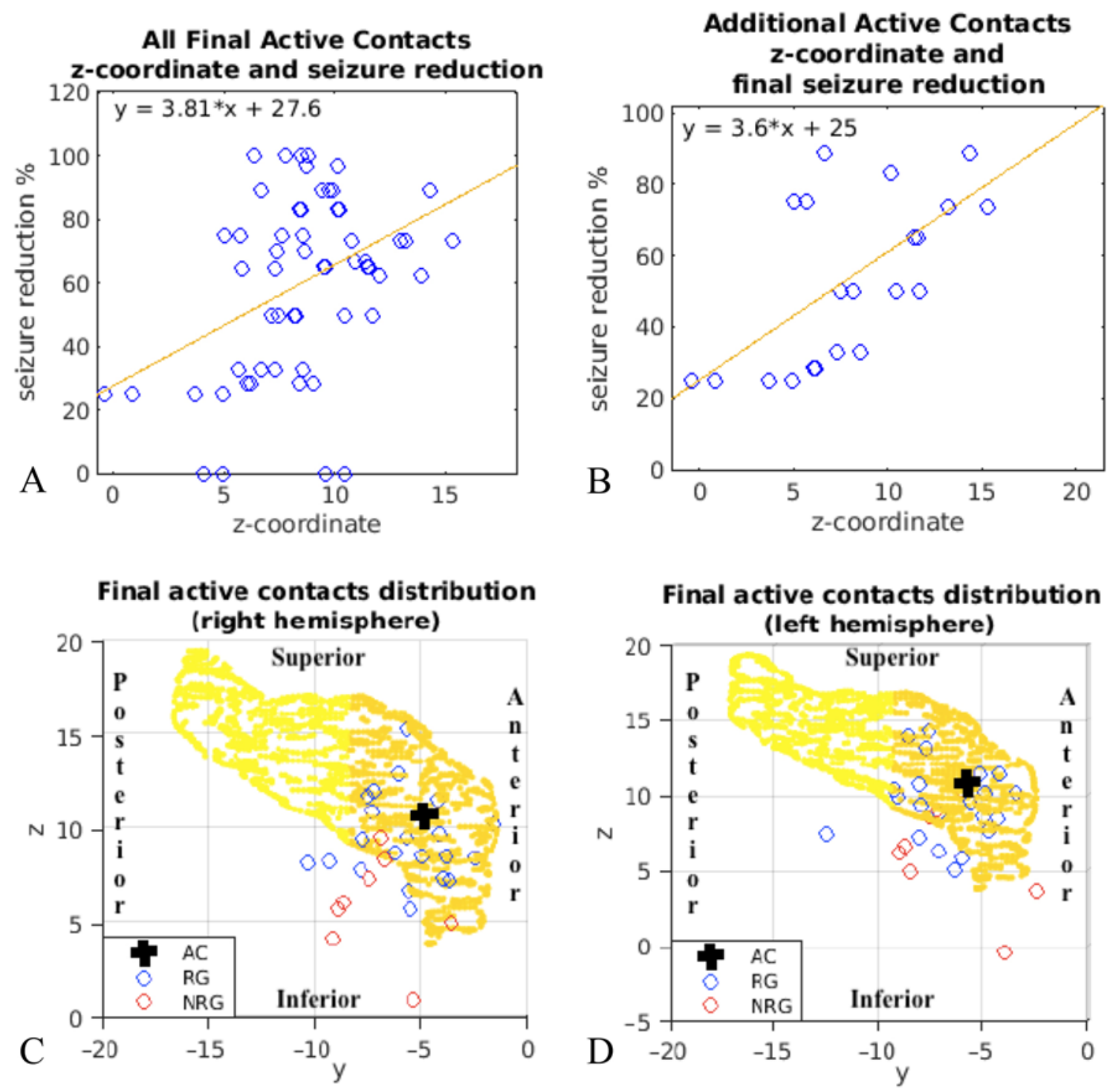

FIG. 3. Distribution and anatomical localization of active contacts in MNI space. A: For all final active contacts, there was a positive linear relationship between the $z$-coordinate and seizure reduction $\left(p<0.01, R^{2}=0.174, r=0.4168\right)$. B: There was a similar linear relationship between the z-coordinate and seizure reduction for the additional active contacts of initial nonresponders ( $p$ $<0.01, R^{2}=0.313, r=0.5598$ ). C: Final active contact distribution with respect to the AC (black cross) in the right hemisphere. The anterior (orange) and posterior (yellow) ATN are indicated. D: Final active contact distribution in the left hemisphere. NRG = nonresponder group; RG = responder group. Figure is available in color online only.

absence of spatial information about DBS contacts in the ATN hinder a hasty conclusion.

Lastly, a posterior parietal extraventricular trajectory, ${ }^{24,25}$ as attempted by the Mayo group, is claimed to have the advantage of accommodating wide contact ( $>2$ leads) in the ATN compared to those with conventional TVA or EVA. However, the study's small sample size limits the ability to assess the robustness of the method and larger studies are warranted.

\section{Consideration of an Optimal Visualization Method for ATN DBS}

A previous study utilizing an ATN-normalized coor- dinate system reported success in patients with more anterior and superior contacts, with responding contacts located at the border between the anterior principal nucleus and anteromedial nucleus of the Schaltenbrand-Wahren atlas. ${ }^{9}$ In addition, modeling the volume of tissue activation presented a more posterior, efficacious target in the Schaltenbrand-Wahren atlas at the inferior and lateral part of the ATN, close to the MTT. ${ }^{3}$ While our results are consistent with previous studies indicating that an optimal target within the ATN is likely, there are variations in the exact proposed target. Both of these previous studies utilized the Schaltenbrand-Wahren atlas, which has been shown to have limitations in the $3 \mathrm{D}$ consistency of sub- 

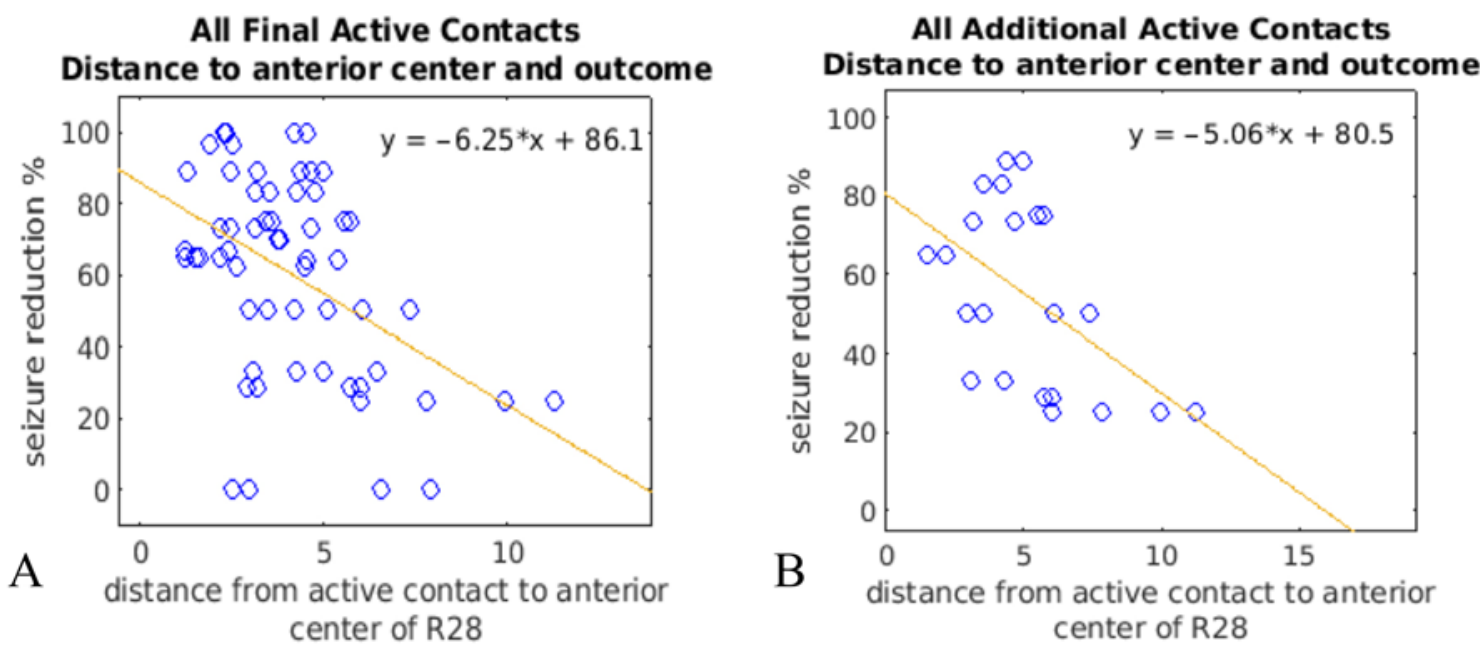

FIG. 4. A: Negative linear relationship between distance from active contact to $A C$ and seizure reduction for all final active contacts $\left(p<0.01, R^{2}=0.217, r=-0.4660\right)$. B: Similar linear relationship between distance and seizure reduction for additional active contacts $\left(p<0.05, R^{2}=0.252, r=-0.5017\right)$. Figure is available in color online only.

cortical structures, likely contributing to variations in the resulting efficacious target. ${ }^{26-28}$ In order to accurately define an optimal target and reduce inconsistencies between patients, we combined nonlinear normalization methods with a consistent atlas.

Lead-DBS, the primary image processing software for our study, is a semiautomated toolbox for lead localization shown to have a near-perfect success rate of lead trajectory reconstruction when accounting for manual correction. ${ }^{13}$ Previous studies have shown that nonlinear models provide greater accuracy over automated linear methods as well as manual registration methods by accommodating differences in brain morphology. ${ }^{29,30}$ Thus, we could obtain an accurate lead localization that used nonlinear registrations to better account for the intersubject morphological variations and allow for convenient intersubject analysis.

To define the ATN, we utilized the DISTAL, a subcortical atlas with high spatial correspondence to the MNI template; the DISTAL utilized the Chakravarty histological atlas, which was based on histological data from Schaltenbrand and Wahren, Gloor and Guberman, and Hirai and Jones. ${ }^{18,20,31-33}$ While we used the DISTAL thalamic parcellation with the Hassler/Schaltenbrand-Wahren nomenclature, there are many other thalamic parcellations available that are not necessarily consistent. ${ }^{19,34}$ Our utilization of nonlinear methods together with the DISTAL allowed for more accurate intersubject analysis in MNI space.
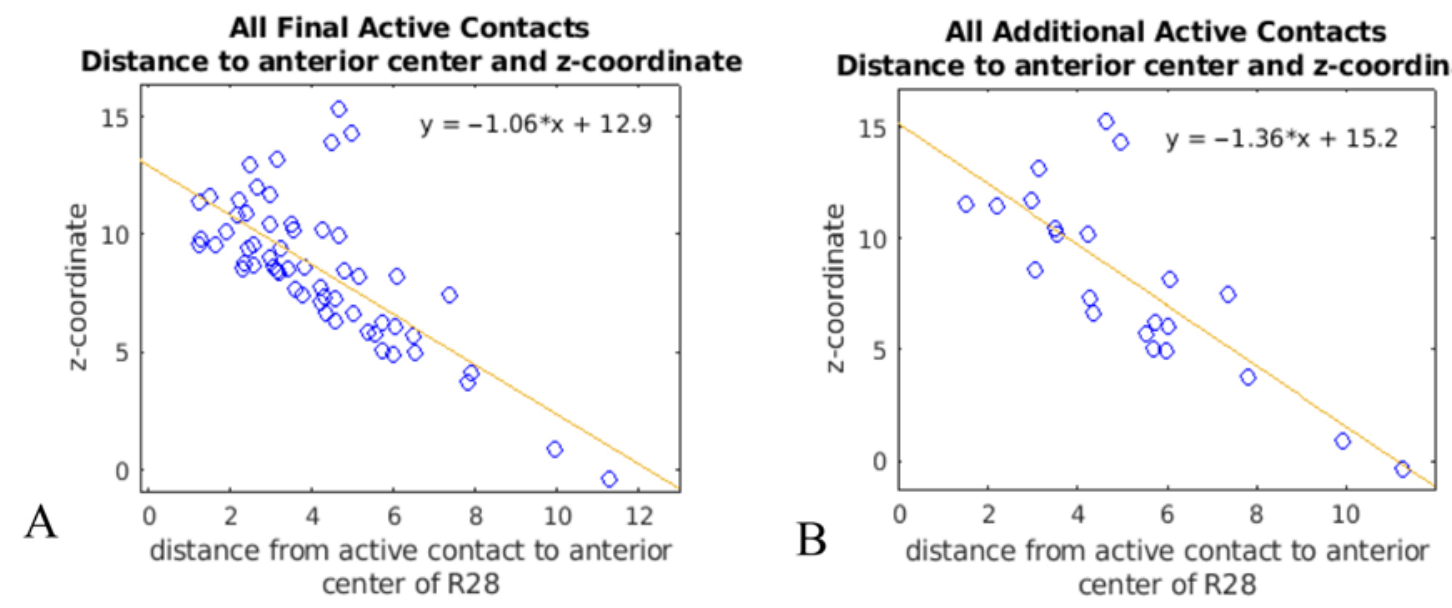

FIG. 5. A: Negative linear relationship between distance from active contact to $A C$ and $z$-coordinate for all final active contacts $(p$ $\left.<0.01, R^{2}=0.533, r=-0.7303\right)$. B: Similar linear relationship between distance and $z$-coordinate for additional active contacts $(p<$ $\left.0.01, R^{2}=0.634, r=-0.7964\right)$. Figure is available in color online only. 


\section{Role of ATN in Epileptic Seizures}

Pharmacological inhibition of the ATN in rats produced anticonvulsant effects and suppressed synchronized EEG activity in seizures, indicating its role in seizure propagation. ${ }^{35,36}$ Furthermore, high-frequency stimulation of the ATN, which likely promotes desynchronization, resulted in anticonvulsant effects. ${ }^{37}$ In addition, the ATN has many other connections from mesial temporal structures and has widespread connections to cortical regions such as the frontal and temporal lobes. ${ }^{38} \mathrm{~A}$ review of previous studies utilizing diffusion tensor imaging (DTI) in DBS revealed better outcomes in patients with electrodes within the DTI target. ${ }^{39}$ Furthermore, extensive bilateral anterior thalamic volume loss was shown to be a characteristic of temporal lobe epilepsy with mesial temporal sclerosis. ${ }^{40}$ As shown, there may be many other factors, including structural changes, that can affect the resulting DBS response and thus the optimal target.

There are some limitations to our study. First, our study only presented results on a small group of patients, and further studies should seek to provide data on a larger patient sample to better define the relationship between active contact location and outcome in ATN DBS. Second, normalization of postoperative CT scans likely resulted in deformations of electrode artifacts, possibly affecting the accuracy of our lead localization analysis. Future studies should seek to utilize atlas-based localization methods that could more precisely define the optimal target. Third, further research is needed to assess other factors, such as degeneration and differences in specific fiber tracts, that contribute to the variations in outcomes.

\section{Conclusions}

Our study demonstrates that the location of active contacts may be closely correlated to the overall seizure reduction outcome. We propose that the optimal target for effective stimulation therapy is the AC (i.e., the center of gravity of the anterior half of the ATN). DBS surgery planning should be customized for each patient in order to accurately target this hot spot. Selective adjustments of lead contact positions are likely to be more effective with the aid of atlas-guided verifications of contact locations.

\section{Acknowledgments}

This work was supported by the National Research Foundation of Korea (NRF) grant funded by the Korean government (Ministry of Science and ICT, grant nos. 2017R1D1A1B03029815, 2018R1D1A1B07048147).

\section{References}

1. Devinsky O, Vezzani A, O’Brien TJ, et al. Epilepsy. Nat Rev Dis Primers. 2018;4:18024.

2. Kerrigan JF, Litt B, Fisher RS, et al. Electrical stimulation of the anterior nucleus of the thalamus for the treatment of intractable epilepsy. Epilepsia. 2004;45(4):346-354.

3. Krishna V, King NK, Sammartino F, et al. Anterior nucleus deep brain stimulation for refractory epilepsy: insights into patterns of seizure control and efficacious target. Neurosurgery. 2016;78(6):802-811.

4. Lee KJ, Shon YM, Cho CB. Long-term outcome of anterior thalamic nucleus stimulation for intractable epilepsy. Stereotact Funct Neurosurg. 2012;90(6):379-385.

5. Osorio I, Overman J, Giftakis J, Wilkinson SB. High frequency thalamic stimulation for inoperable mesial temporal epilepsy. Epilepsia. 2007;48(8):1561-1571.

6. Kim SH, Lim SC, Kim J, et al. Long-term follow-up of anterior thalamic deep brain stimulation in epilepsy: a 11-year, single center experience. Seizure. 2017;52:154-161.

7. Oh YS, Kim HJ, Lee KJ, et al. Cognitive improvement after long-term electrical stimulation of bilateral anterior thalamic nucleus in refractory epilepsy patients. Seizure. 2012;21(3):183-187.

8. Möttönen T, Katisko J, Haapasalo J, et al. Defining the anterior nucleus of the thalamus (ANT) as a deep brain stimulation target in refractory epilepsy: delineation using 3 T MRI and intraoperative microelectrode recording. Neuroimage Clin. 2015:7:823-829.

9. Lehtimäki K, Möttönen T, Järventausta K, et al. Outcome based definition of the anterior thalamic deep brain stimulation target in refractory epilepsy. Brain Stimul. 2016;9(2):268-275.

10. Cukiert A, Lehtimäki K. Deep brain stimulation targeting in refractory epilepsy. Epilepsia. 2017;58(suppl 1):80-84.

11. Kim SH, Son BC, Lim SC, et al. EEG driving response during low-frequency stimulation of anterior thalamic nucleus: is it a good predictor of the correct location of DBS electrode? Clin Neurophysiol. 2014;125(5):1065-1066.

12. Fisher RS, Velasco AL. Electrical brain stimulation for epilepsy. Nat Rev Neurol. 2014;10(5):261-270.

13. Horn A, Kühn AA. Lead-DBS: a toolbox for deep brain stimulation electrode localizations and visualizations. Neuroimage. 2015;107:127-135.

14. Avants BB, Epstein CL, Grossman M, Gee JC. Symmetric diffeomorphic image registration with cross-correlation: evaluating automated labeling of elderly and neurodegenerative brain. Med Image Anal. 2008;12(1):26-41.

15. Fonov V, Evans AC, Botteron K, et al. Unbiased average age-appropriate atlases for pediatric studies. Neuroimage. 2011;54(1):313-327.

16. Schönecker T, Kupsch A, Kühn AA, et al. Automated optimization of subcortical cerebral MR imaging-atlas coregistration for improved postoperative electrode localization in deep brain stimulation. AJNR Am J Neuroradiol. 2009;30(10):1914-1921.

17. Husch A, V Petersen M, Gemmar P, et al. PaCER - a fully automated method for electrode trajectory and contact reconstruction in deep brain stimulation. Neuroimage Clin. 2017;17:80-89.

18. Ewert S, Plettig P, Li N, et al. Toward defining deep brain stimulation targets in MNI space: a subcortical atlas based on multimodal MRI, histology and structural connectivity. Neuroimage. 2018;170:271-282.

19. Hassler R. Architectonic organization of the thalamic nuclei. In: Schaltenbrand G, ed. Stereotaxy of the Human Brain. Georg Thieme Verlag; 1977.

20. Chakravarty MM, Bertrand G, Hodge CP, et al. The creation of a brain atlas for image guided neurosurgery using serial histological data. Neuroimage. 2006;30(2):359-376.

21. Koeppen JA, Nahravani F, Kramer M, et al. Electrical stimulation of the anterior thalamus for epilepsy: clinical outcome and analysis of efficient target. Neuromodulation. 2019;22(4):465-471.

22. Herrman H, Egge A, Konglund AE, et al. Anterior thalamic deep brain stimulation in refractory epilepsy: a randomized, double-blinded study. Acta Neurol Scand. 2019;139(3):294304.

23. Lehtimäki K, Coenen VA, Gonçalves Ferreira A, et al. The surgical approach to the anterior nucleus of thalamus in patients with refractory epilepsy: experience from the In- 
ternational Multicenter Registry (MORE). Neurosurgery. 2019;84(1):141-150.

24. Van Gompel JJ, Klassen BT, Worrell GA, et al. Anterior nuclear deep brain stimulation guided by concordant hippocampal recording. Neurosurg Focus. 2015;38(6):E9.

25. Wang YC, Grewal SS, Middlebrooks EH, et al. Targeting analysis of a novel parietal approach for deep brain stimulation of the anterior nucleus of the thalamus for epilepsy. Epilepsy Res. 2019;153:1-6.

26. Nowinski WL, Liu J, Arumugam T. Quantification and visualization of three-dimensional inconsistency of the globus pallidus internus in the Schaltenbrand-Wahren brain atlas. Stereotact Funct Neurosurg. 2006;84(5-6):236-242.

27. Nowinski WL, Liu J, Thirunavuukarasuu A. Quantification and visualization of the three-dimensional inconsistency of the subthalamic nucleus in the Schaltenbrand-Wahren brain atlas. Stereotact Funct Neurosurg. 2006;84(1):46-55.

28. Nowinski WL, Liu J, Thirunavuukarasuu A. Quantification and visualization of three-dimensional inconsistency of the ventrointermediate nucleus of the thalamus in the Schaltenbrand-Wahren brain atlas. Acta Neurochir (Wien). 2008;150(7):647-653.

29. Harston GW, Minks D, Sheerin F, et al. Optimizing image registration and infarct definition in stroke research. Ann Clin Transl Neurol. 2017;4(3):166-174.

30. Woods RP, Grafton ST, Watson JD, et al. Automated image registration: II. Intersubject validation of linear and nonlinear models. J Comput Assist Tomogr. 1998;22(1):153-165.

31. Gloor P, Guberman AH. The temporal lobe \& limbic system. CMAJ. 1997;157:1597-1598.

32. Hirai T, Jones EG. A new parcellation of the human thalamus on the basis of histochemical staining. Brain Res Brain Res Rev. 1989;14(1):1-34.

33. Schaltenbrand G, Wahren W. Atlas for Stereotaxy of the Human Brain. Georg Thieme Verlag; 1977.

34. Mai JK, Majtanik M. Toward a common terminology for the thalamus. Front Neuroanat. 2019;12:114.

35. Mirski MA, Ferrendelli JA. Anterior thalamic mediation of generalized pentylenetetrazol seizures. Brain Res. 1986;399(2):212-223.
36. Mirski MA, McKeon AC, Ferrendelli JA. Anterior thalamus and substantia nigra: two distinct structures mediating experimental generalized seizures. Brain Res. 1986;397(2):377_ 380 .

37. Mirski MA, Rossell LA, Terry JB, Fisher RS. Anticonvulsant effect of anterior thalamic high frequency electrical stimulation in the rat. Epilepsy Res. 1997;28(2):89-100.

38. Le Gros Clark WE, Boggon RH. On the connections of the anterior nucleus of the thalamus. J Anat. 1933;67(Pt 2):215226.9 .

39. See AAQ, King NKK. Improving surgical outcome using diffusion tensor imaging techniques in deep brain stimulation. Front Surg. 2017;4:54.

40. Mueller SG, Laxer KD, Barakos J, et al. Involvement of the thalamocortical network in TLE with and without mesiotemporal sclerosis. Epilepsia. 2010;51(8):1436-1445.

\section{Disclosures}

The authors report no conflict of interest concerning the materials or methods used in this study or the findings specified in this paper.

\section{Author Contributions}

Conception and design: Shon, Guo, Koo, Bhadelia. Acquisition of data: Shon, Seo, Hong, Joo, S Lee, JI Lee, Cho. Analysis and interpretation of data: Shon, Guo, Koo, Kim, S Lee. Drafting the article: Guo. Critically revising the article: Shon, Koo, Kim, Bhadelia. Reviewed submitted version of manuscript: Shon. Statistical analysis: Guo. Administrative/technical/material support: Shon. Study supervision: Shon, Guo, Koo, Kim, Bhadelia, Seo, JI Lee.

\section{Correspondence}

Young-Min Shon: Samsung Medical Center, Sungkyunkwan University School of Medicine, Seoul, Korea. youngmin.shon@ samsung.com. 